UDC 539.3

\title{
Unloading wave in the cylindrical network from nonlinear elastic fibers
}

\author{
Mexseti A. Rustamova \\ Institute of Mathematics and Mechanics of National Academy of Sciences of Azerbaijan, 9 B. Vahabzadeh St., Baku, AZ 1143, Republic of Azerbaijan
}

Received: January 30, 2019

Revised: February 27, 2019

Accepted: March 16, 2019

\section{Keywords:}

nonlinear elastic fibers;

wave of unloading;

cylindrical network;

continuous waves
Abstract

Aims of research. Investigation of a wave of unloading in a cylindrical network of nonlinear elastic fibers. Given the many options for wave propagation in cylindrical networks, an attempt is made to solve the problem of continuous waves.

Methods. The movement of the network in the axial direction is considered. To a basis of a cylindrical system are accepted: an individual vector $\vec{i}$ parallel to a cylinder axis, $\vec{j}-$ an individual vector of a tangent to cross-section section of the cylinder, $\vec{k}-$ an individual vector perpendicular to the previous ones, $x-$ is the coordinate in the direction of the axis of the cylinder, $y-$ is the length of an arc of the circumference of the cylinder. The problem reduces to a hyperbolic system of equations under appropriate conditions. Since the wave speed increases when the net is stretched, the stretch wave will obviously be discontinuous. In order to study continuous waves, the problem of wave propagation is solved when unloading a pre-stretched cylinder from a nonlinear basis. The problem is solved by the method of characteristics.

Results. The results are illustrated with calculations and can be used at calculations of various flexible pipes, including flexible drilling.

\section{Introduction}

The equation of movement of [1] networks in space has a form, constructed on the basis of the theory of Rahmatullin. In articles [2-7] waves in networks in rectangular Cartesian system of coordinates were investigated. Here waves in a cylindrical system of co-ordinates are investigated. Obviously, during stretching a cylindrical network is going to be narrowed. Being placed on a rigid pipe during motion, it will be exposed to operate a force of a friction between it and a pipe. In order to avoid it, the network is replaced on a screw pipe of a special profile. Such pipes are applied, in particular at the process of drilling of chinks. In practice, these phenomena can take place in the flexible pipelines.

Aim is research of waves in cylindrical sets. Considering sets of variants of distribution of waves

(C) Rustamova M.A., 2019

This work is licensed under a Creative Commons Attribution 4.0 International License in cylindrical networks, it is attempted to solve a problem about continuous waves.

\section{The general equations of movement of a network}

The equation of motion of the network, taking into account the reaction of the supporting body and the geometric relations will have the form, in contrast to [2].

$$
\begin{aligned}
& \frac{\partial}{\partial s_{1}}\left(\sigma_{1} \vec{\tau}_{1}\right)+\frac{\partial}{\partial s_{2}}\left(\sigma_{2} \vec{\tau}_{2}\right)=\left(\rho_{1}+\rho_{2}\right) \frac{\partial^{2} \vec{r}}{\partial t^{2}}+p \vec{n} ; \\
& \left(1+e_{1}\right) \vec{\tau}_{1}=\frac{\partial \vec{r}}{\partial s_{1}} ;\left(1+e_{2}\right) \vec{\tau}_{2}=\frac{\partial \vec{r}}{\partial s_{2}} .
\end{aligned}
$$

Here, $\vec{r}$ - radius vector of a particle of a network; $p$ - power of a reaction of the cylinder; $e_{1}, e_{2}$ - the relative lengthening, corresponding threads; 
$s_{1}, s_{2}$ - Lagranzhevy of co-ordinates of particles of threads; $\sigma_{1}$ and $\sigma_{2}$ the conditional pressure defined as the sum of tension of separate threads of one family (crossing a site of a thread of other family), carried to an initial length of a considered element.

Such distribution of weight and efforts is admissible at sufficient dense network, $\rho_{1}+\rho_{2}-$ weights of elements of the network, having corresponding directions on area unit in an initial condition, $\tau_{1}, \tau_{2}$ individual vectors tangents to threads, $\vec{n}-$ a normal to a surface of the cylindrical basis.

\section{Coordinate system}

To a basis of a cylindrical system are accepted (figure 1): an individual vector $\vec{i}$ parallel to a cylinder axis, $\vec{j}$ an individual vector of a tangent to crosssection section of the cylinder, $\vec{k}$ an individual vector perpendicular to the previous ones, $x-$ is the coordinate in the direction of the axis of the cylinder, $y$ - is the length of an arc of the circumference of the cylinder. Then

$\vec{\tau}_{1}=\cos \gamma_{1} \vec{i}+\sin \gamma_{1} \vec{j} ; \vec{\tau}_{2}=\cos \gamma_{2} \vec{i}+\sin \gamma_{2} \vec{j}$,

where $\gamma_{1,2}$ - corners of threads formed with a cylinder axis.

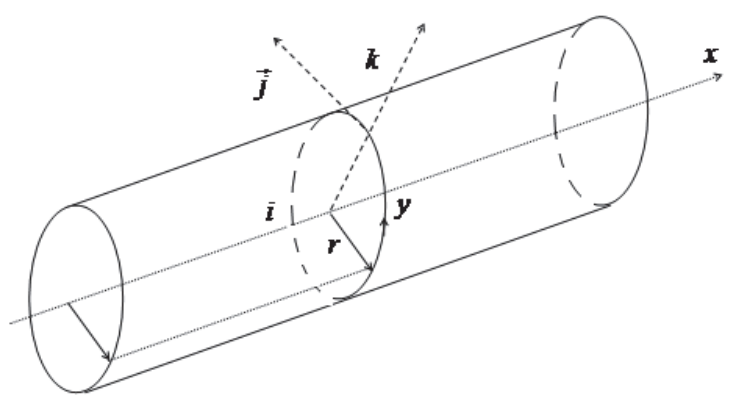

Figure 1. Coordinate system

Derivatives:

$$
\begin{aligned}
& \frac{\partial \vec{\tau}_{1}}{\partial s_{1}}=\cos \gamma_{1} \frac{\partial \vec{i}}{\partial s_{1}}+\vec{i} \frac{\partial\left(\cos \gamma_{1}\right)}{\partial s_{1}}+ \\
& +\sin \gamma_{1} \frac{\partial \vec{j}}{\partial s_{1}}+\vec{j} \frac{\partial\left(\sin \gamma_{1}\right)}{\partial s_{1}} ;
\end{aligned}
$$

$$
\begin{aligned}
& \frac{\partial \vec{\tau}_{2}}{\partial s_{2}}=\cos \gamma_{2} \frac{\partial \vec{i}}{\partial s_{2}}+\vec{i} \frac{\partial\left(\cos \gamma_{2}\right)}{\partial s_{2}}+ \\
& +\sin \gamma_{2} \frac{\partial \vec{j}}{\partial s_{2}}+\vec{j} \frac{\partial\left(\sin \gamma_{2}\right)}{\partial s_{2}} .
\end{aligned}
$$

Considering

$$
\begin{aligned}
& \frac{\partial \vec{i}}{\partial s_{1}}=\frac{\partial \vec{i}}{\partial s_{2}}=0 ; \quad \frac{\partial \vec{j}}{\partial s_{1}}=-\frac{\sin \gamma_{1}}{r} \vec{k} ; \\
& \frac{\partial \vec{j}}{\partial s_{2}}=-\frac{\sin \gamma_{2}}{r} \vec{k} .
\end{aligned}
$$

From (3) we will get

$$
\begin{aligned}
& \frac{\partial \vec{\tau}_{1}}{\partial s_{1}}=\frac{\partial\left(\cos \gamma_{1}\right)}{\partial s_{1}} \vec{i}-\frac{\left(\sin \gamma_{1}\right)^{2}}{r} \vec{k}+\frac{\partial\left(\sin \gamma_{1}\right)}{\partial s_{1}} \vec{j} . \\
& \frac{\partial \vec{\tau}_{2}}{\partial s_{2}}=\frac{\partial\left(\cos \gamma_{2}\right)}{\partial s_{2}} \vec{i}-\frac{\left(\sin \gamma_{2}\right)^{2}}{r} \vec{k}+\frac{\partial\left(\sin \gamma_{2}\right)}{\partial s_{2}} \vec{j} .
\end{aligned}
$$

Also considering $\vec{r}=x \vec{i}+r \vec{k}$ we have

$$
\begin{aligned}
& \frac{\partial \vec{r}}{\partial t}=\frac{\partial x}{\partial t} \vec{i}+r \frac{\partial \vec{k}}{\partial t}=\frac{\partial x}{\partial t} \vec{i}+r \omega \vec{j} \\
& \frac{\partial^{2} \vec{r}}{\partial t^{2}}=\frac{\partial^{2} x}{\partial t^{2}} \vec{i}+r \frac{\partial \omega}{\partial t} \vec{j}+r \omega \frac{\partial \vec{j}}{\partial t}
\end{aligned}
$$

or

$$
\frac{\partial^{2} \vec{r}}{\partial t^{2}}=\frac{\partial^{2} x}{\partial t^{2}} \vec{i}+r \varepsilon \vec{j}+r \omega^{2} \vec{k}
$$

where $\omega$-angular speed; $\varepsilon$ - angular acceleration.

\section{The equations of movement of a cylindrical network}

Having substituted (4) and (5) in (1) we will get

$$
\begin{aligned}
& \frac{\partial}{\partial s_{1}}\left(\sigma_{1} \cos \gamma_{1}\right) \vec{i}-\frac{\sigma_{1}}{r}\left(\sin \gamma_{1}\right)^{2} \vec{k}+\frac{\partial}{\partial s_{1}}\left(\sigma_{1} \sin \gamma_{1}\right) \vec{j}+ \\
& +\frac{\partial}{\partial s_{2}}\left(\sigma_{2} \cos \gamma_{2}\right) \vec{i}-\frac{\sigma_{2}}{r}\left(\sin \gamma_{2}\right)^{2} \vec{k}+\frac{\partial}{\partial s_{2}}\left(\sigma_{2} \sin \gamma_{2}\right) \vec{j}= \\
& =\left(\rho_{1}+\rho_{2}\right) \frac{\partial^{2} x}{\partial t^{2}} \vec{i}+\left(\rho_{1}+\rho_{2}\right) r \varepsilon \vec{j}+\left(\rho_{1}+\rho_{2}\right) r \omega^{2} \vec{k}+p \vec{n} ;
\end{aligned}
$$


$\frac{\partial}{\partial s_{1}}\left(\sigma_{1} \cos \gamma_{1}\right)+\frac{\partial}{\partial s_{2}}\left(\sigma_{2} \cos \gamma_{2}\right)=\left(\rho_{1}+\rho_{2}\right) \frac{\partial^{2} x}{\partial t^{2}}$

$\frac{\partial}{\partial s_{1}}\left(\sigma_{1} \sin \gamma_{1}\right)+\frac{\partial}{\partial s_{2}}\left(\sigma_{2} \sin \gamma_{2}\right)=\left(\rho_{1}+\rho_{2}\right) r \varepsilon$

$-\frac{\sigma_{1}}{r}\left(\sin \gamma_{1}\right)^{2}-\frac{\sigma_{2}}{r}\left(\sin \gamma_{2}\right)^{2}=\left(\rho_{1}+\rho_{2}\right) r \omega^{2}+p$.

Next is the symmetrical arrangement of the right and left fibers. Then the equations (6), considering $\sigma_{1}=\sigma_{2}=\sigma, \quad \gamma_{1}=-\gamma_{2}=\gamma, \omega=0, \varepsilon=0$ will become:

$2 \frac{\partial}{\partial s}(\sigma \cos \gamma)=\left(\rho_{1}+\rho_{2}\right) \frac{\partial^{2} x}{\partial t^{2}}$

$2 \frac{\sigma}{r}(\sin \gamma)^{2}=-p$

\section{Geometrical correlations}

Let's define a derivative of a radius-vector $\vec{r}$ with respect to $s$. Having designated $\vec{r}=x \vec{i}+r \vec{k}$.

$\frac{\partial \vec{r}}{\partial s}=\frac{\partial x}{\partial s} \vec{i}+\frac{\partial k}{\partial s} r=\frac{\partial x}{\partial s} \vec{i}+\frac{\partial y}{\partial s} \vec{j}$.

Where according to (1) and (3)

$\left(1+e_{1}\right) \cos \gamma_{1} \vec{i}+\left(1+e_{1}\right) \sin \gamma_{1} \vec{j}=\frac{\partial r}{\partial s_{1}}$

$\left(1+e_{2}\right) \cos \gamma_{2} \vec{i}+\left(1+e_{2}\right) \sin \gamma_{2} \vec{j}=\frac{\partial r}{\partial s_{2}}$

$(1+e) \cos \gamma=\frac{\partial x}{\partial s}$

$(1+e) \sin \gamma=\frac{\partial y}{\partial s}$.

As the network does not rotate, then $y=$ const.

$$
\frac{\partial((1+e) \sin \gamma)}{\partial t}=0
$$

or

$$
\left(1+\mathrm{e}_{0}\right) \sin \gamma_{0}=(1+\mathrm{e}) \sin \gamma,
$$

where $e_{0}$ also $\gamma_{0}$ are values of parameters in an initial condition.

Using (8) of the first equation (6) it is possible to write:

$\frac{\partial \sigma}{\partial s} \frac{1}{1+e} \frac{\partial x}{\partial s}+\sigma \frac{\partial}{\partial s}\left(\frac{1}{1+e} \frac{\partial x}{\partial s}\right)=\left(\rho_{1}+\rho_{2}\right) \frac{\partial^{2} x}{\partial t^{2}} ;$

$\frac{1}{1+e} \frac{\partial \sigma}{\partial s} \frac{\partial x}{\partial s}-\sigma \frac{1}{(1+e)^{2}} \frac{\partial e}{\partial s} \frac{\partial x}{\partial s}+\frac{\sigma}{1+e} \frac{\partial^{2} x}{\partial s^{2}}=$

$=\left(\rho_{1}+\rho_{2}\right) \frac{\partial^{2} x}{\partial t^{2}}$

$\frac{\sigma^{\prime}}{1+e} \frac{\partial e}{\partial s} \frac{\partial x}{\partial s}-\sigma \frac{1}{(1+e)^{2}} \frac{\partial e}{\partial s} \frac{\partial x}{\partial s}+\frac{\sigma}{1+e} \frac{\partial^{2} x}{\partial s^{2}}=$

$=\left(\rho_{1}+\rho_{2}\right) \frac{\partial^{2} x}{\partial t^{2}} ;$

$\left(\frac{\sigma^{\prime}}{1+\mathrm{e}}-\frac{\sigma}{(1+e)^{2}}\right) \frac{1}{1+e} \frac{\partial^{2} x}{\partial s^{2}}\left(\frac{\partial x}{\partial s}\right)^{2}+\frac{\sigma}{1+e} \frac{\partial^{2} x}{\partial s^{2}}=$

$=\left(\rho_{1}+\rho_{2}\right) \frac{\partial^{2} x}{\partial t^{2}}$.

From (11) we will get the following equation:

$$
\begin{aligned}
& {\left[\left(\frac{\sigma^{\prime}}{(1+e)^{2}}-\frac{\sigma}{(1+e)^{3}}\right)\left(\frac{\partial x}{\partial s}\right)^{2}+\frac{\sigma}{1+e}\right] \frac{\partial^{2} x}{\partial s^{2}}=} \\
& =\left(\rho_{1}+\rho_{2}\right) \frac{\partial^{2} x}{\partial t^{2}}
\end{aligned}
$$

Last equation represents quasilinear equation in partial derivatives.

$$
\begin{aligned}
& a=\sqrt{\left(\frac{\sigma^{\prime}}{(1+e)^{2}}-\frac{\sigma}{(1+e)^{3}}\right)\left(\frac{\partial x}{\partial s}\right)^{2}+\frac{\sigma}{1+e}} . \\
& \text { Here } \quad e=\sqrt{\left(\frac{\partial x}{\partial s}\right)^{2}+\left(1+e_{0}\right) \operatorname{sim}_{0}-1} ; \quad \varepsilon=\frac{\partial x}{\partial s} ;
\end{aligned}
$$
$\sigma\left(\frac{\partial x}{\partial s}\right)$ it is set.

If we take $\sigma, \sigma^{\prime}$ in the following way $\sigma=\alpha\left(\frac{\partial x}{\partial s}\right)^{2} ; \sigma^{\prime}=2 \alpha\left(\frac{\partial x}{\partial s}\right)$, we get the above given plot. 


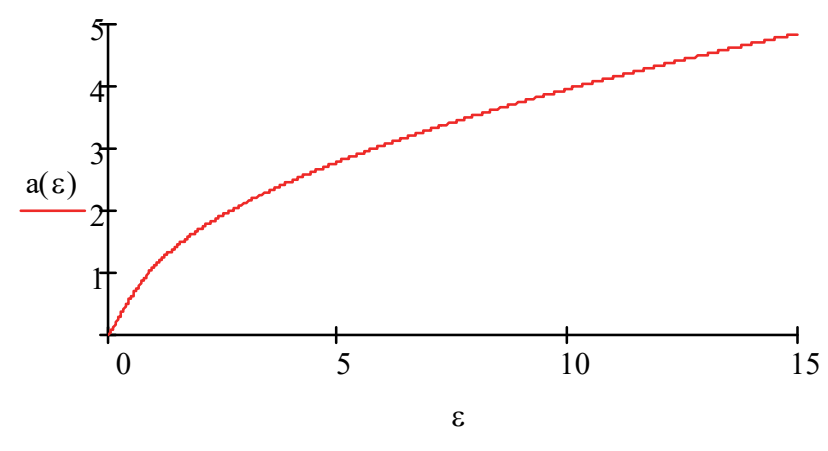

Figure 2. The graph for dependence between quantities $\varepsilon$ and $a(\varepsilon)$

Let's consider another case.

Flat nonlinear elastic, in other words, $\sigma=\alpha_{1} \cdot \frac{\partial x}{\partial s}+\alpha_{2}\left(\frac{\partial x}{\partial s}\right)^{2}$, system (8), (9) and (10) can be reduced to one quasilinear equation of the second order.

From (6) follows

$2 \cdot \frac{\partial}{\partial S}(\sigma \cos \gamma)=\left(\rho_{1}+\rho_{2}\right) \cdot \frac{\partial^{2} x}{\partial t^{2}}$

$2 \cdot \frac{\partial}{\partial S}\left(\alpha_{1} \cdot \frac{\partial x}{\partial s}+\alpha_{2}\left(\frac{\partial x}{\partial s}\right)^{2}\right) \cos \gamma=\left(\rho_{1}+\rho_{2}\right) \cdot \frac{\partial^{2} x}{\partial t^{2}}$

$2 \cdot \frac{\partial}{\partial S}\left(\alpha_{1} \cdot \cos \gamma \frac{\partial x}{\partial s}\right)+2 \cdot \frac{\partial}{\partial S}\left(\alpha_{2} \cdot \cos \gamma\left(\frac{\partial x}{\partial s}\right)^{2}\right)=$

$=\left(\rho_{1}+\rho_{2}\right) \cdot \frac{\partial^{2} x}{\partial t^{2}}$

$2 \cdot \alpha_{1} \frac{\partial x}{\partial S} \frac{\partial \cos \gamma}{\partial s}+2 \cdot \alpha_{1} \cos \gamma \frac{\partial^{2} x}{\partial s^{2}}+$

$+2 \cdot \alpha_{2}\left(\frac{\partial x}{\partial s}\right)^{2} \frac{\partial \cos \gamma}{\partial S}+4 \cdot \alpha_{2} \cos \gamma \frac{\partial x}{\partial s} \frac{\partial^{2} x}{\partial s^{2}}=$

$=\left(\rho_{1}+\rho_{2}\right) \cdot \frac{\partial^{2} x}{\partial t^{2}}$.

From (8)

$\cos \gamma=\frac{1}{1+e} \frac{\partial x}{\partial s}$

$\frac{\partial \cos \gamma}{\partial S}=-\frac{1}{(1+e)^{2}} \frac{\partial e}{\partial s} \frac{\partial x}{\partial s}+\frac{1}{(1+e)} \frac{\partial^{2} x}{\partial s^{2}}$.
From (8) and (10)

$$
\begin{aligned}
& (1+e)^{2} \cos ^{2} \gamma=\frac{\partial^{2} x}{\partial s^{2}} \\
& \left(1+e_{0}\right)^{2} \sin ^{2} \gamma_{0}=(1+e)^{2} \sin ^{2} \gamma \\
& \frac{\partial^{2} x}{\partial S^{2}}+\left(1+e_{0}\right)^{2} \sin ^{2} \gamma_{0}=(1+e)^{2}
\end{aligned}
$$

From (17)

$2(1+e) \frac{\partial e}{\partial s}=2 \frac{\partial x}{\partial s} \frac{\partial^{2} x}{\partial s^{2}}$

or

$\frac{\partial e}{\partial s}=\frac{1}{(1+e)} \frac{\partial x}{\partial s} \frac{\partial^{2} x}{\partial s^{2}}$.

Using (15), (16) and (18) in (14) we will get

$\alpha_{1}\left(\frac{\partial x}{\partial s}\left(-\frac{1}{(1+e)^{3}}\left(\frac{\partial x}{\partial s}\right)^{2} \frac{\partial^{2} x}{\partial s^{2}}+\frac{1}{1+e} \frac{\partial^{2} x}{\partial s^{2}}\right)+\frac{1}{1+e} \frac{\partial x}{\partial s} \frac{\partial^{2} x}{\partial s^{2}}\right)+$

$+\alpha_{2}\left(\left(\frac{\partial x}{\partial s}\right)^{2}\left(-\frac{1}{(1+e)^{3}}\left(\frac{\partial x}{\partial s}\right)^{2} \frac{\partial^{2} x}{\partial s^{2}}+\frac{1}{1+e} \frac{\partial^{2} x}{\partial s^{2}}\right)+\frac{2}{1+e} \frac{\partial x}{\partial s} \frac{\partial x}{\partial s} \frac{\partial^{2} x}{\partial s^{2}}\right)=$

$=\frac{\left(\rho_{1}+\rho_{2}\right)}{2} \cdot \frac{\partial^{2} x}{\partial t^{2}}$;

$\alpha_{1}\left(-\frac{1}{(1+e)^{3}}\left(\frac{\partial x}{\partial s}\right)^{3} \frac{\partial^{2} x}{\partial s^{2}}+\frac{2}{1+e} \frac{\partial x}{\partial s} \frac{\partial^{2} x}{\partial s^{2}}\right)+$

$+\alpha_{2}\left(-\frac{1}{(1+e)^{3}}\left(\frac{\partial x}{\partial s}\right)^{4} \frac{\partial^{2} x}{\partial s^{2}}+\frac{3}{1+e}\left(\frac{\partial x}{\partial s}\right)^{2} \frac{\partial^{2} x}{\partial s^{2}}\right)=$

$=\frac{\left(\rho_{1}+\rho_{2}\right)}{2} \cdot \frac{\partial^{2} x}{\partial t^{2}}$.

Here, $a_{0}^{2}=\frac{2}{\rho_{1}+\rho_{2}}$.

Last equation can be represented in the above given form:

$\left[-\frac{1}{(1+e)^{3}}\left(\alpha_{1}+\alpha_{2} \frac{\partial x}{\partial s}\right)\left(\frac{\partial x}{\partial s}\right)^{3}+\left(\frac{1}{(1+e)}\left(2 \alpha_{1}+3 \alpha_{2} \frac{\partial x}{\partial s}\right) \frac{\partial x}{\partial s}\right)\right] \frac{\partial^{2} x}{\partial s^{2}}=$ $=\frac{\left(\rho_{1}+\rho_{2}\right)}{2} \cdot \frac{\partial^{2} x}{\partial t^{2}}$.

$a\left(\frac{\partial x}{\partial s}\right) \frac{\partial^{2} x}{\partial s^{2}}=a_{0} \cdot \frac{\partial^{2} x}{\partial t^{2}}$.

The last equation is a quasilinear partial differential equation. 
The coefficient at $\frac{\partial^{2} x}{\partial s^{2}}$ in (19) increases with the growth of $\frac{\partial x}{\partial s}$, therefore speed of waves with deformation growth increases, conducts to the formation of shock waves [8].

Continuous waves will occur when unloading a pre-stretched cylinder. Here, too, the method of characteristics is used (figure 3).

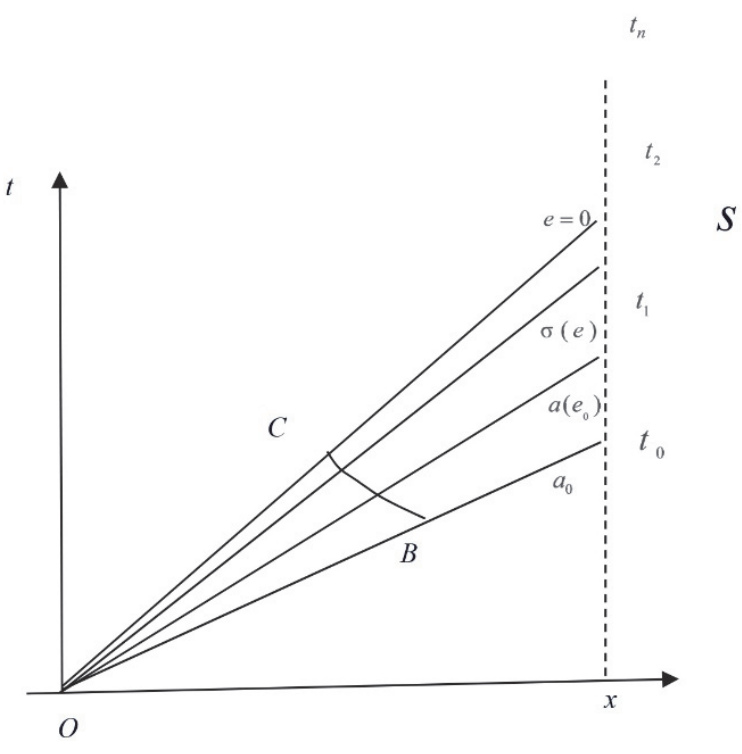

Figure 3. The method of characteristics

From a point 0 wave extends with the maximum speed $a\left(e_{0}\right)$ as waves with smaller deformation extend with smaller speed and will not influence a condition at the front.

Let the cylinder to locate in the stretched condition $e_{0}$.

On border the cylinder unloads, in other words, its end moves with a speed of $\vartheta$.

Characteristics of the equation (19) have a form:

$d s=a d t$

$d s=-a d t$

Conditions on characteristics

$d x_{t}=a d x_{s}\left(\frac{\partial x}{\partial s}=x_{s}, \quad \frac{\partial x}{\partial t}=x_{t}\right)$ and

$$
d x_{t}=-a d x_{s}
$$

$$
a=a_{0} \sqrt{\left[-\frac{1}{(1+e)^{3}}\left(\alpha_{1}+\alpha_{2} \frac{\partial x}{\partial s}\right)\left(\frac{\partial x}{\partial s}\right)^{3}+\left(\frac{1}{(1+e)}\left(2 \alpha_{1}+3 \alpha_{2} \frac{\partial x}{\partial s}\right) \frac{\partial x}{\partial s}\right)\right]} .
$$

The front of an unloading wave moves with a speed $a\left(e_{0}\right)$. In the field of SOA (figure 2) a rest condition. From a condition on negative characteristic BC follows $x_{t}=-\int_{x_{s}^{0}}^{x_{s}} a d x_{s}$; differentiating in a direction of the positive characteristic we have $d x_{t}=-a d x_{s}$.

Comparing with (22) we get $x_{t}=$ const, $x_{s}=$ const. In other words, on positive characteristics $x_{t}, x_{s}$ are constant.

From (20) we have, considering a constancy $x_{s}$ on the characteristic

$$
x=a\left(t-t_{0}\right) .
$$

At $x=0$ we choose $t_{0}$ and define $\varepsilon$.

From (24)

$t_{0}=t-\frac{x}{a}$

and accordingly

$\vartheta=\vartheta_{0}\left(t_{0}\right)$

$\vartheta=\vartheta_{0}\left(t-\frac{x}{a}\right)$

Let's consider an example: $\gamma_{0}=\frac{\pi}{4} \quad$ and $\gamma_{0}=\frac{\pi}{6}, \quad e_{0}=0,1 ; \quad a_{0}=5000 \mathrm{M} / \mathrm{c}$.

The plot of $\left(a\left(x_{s}\right)=a(\varepsilon)\left(x_{s}=\varepsilon\right), f(\varepsilon)\right.$ is shown on figure 3 and the plot of $p(\varepsilon), m(\varepsilon)$ is manifested on figure 4 . 


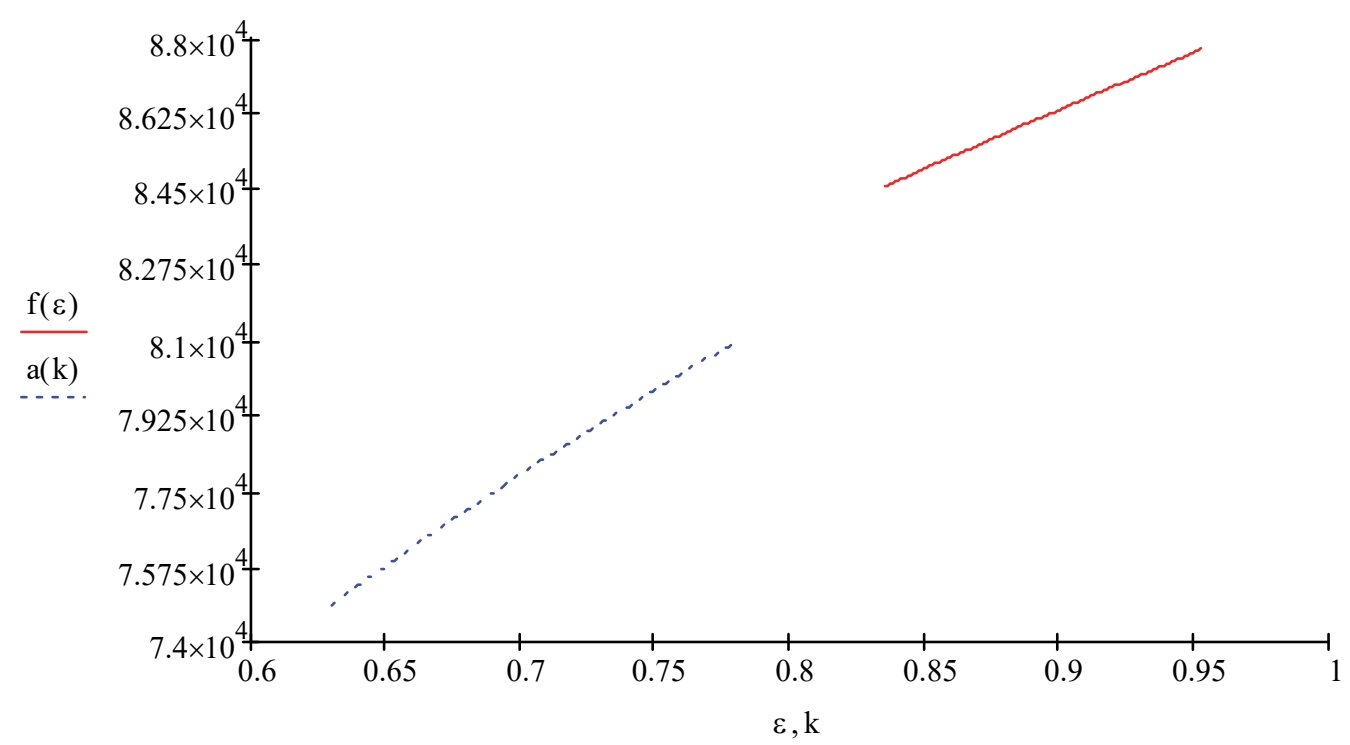

Figure. 4 The graph for dependence between quantities $\varepsilon, k$ and $f(\varepsilon), a(k)$ :

$$
\left(\gamma_{0}=\frac{\pi}{4} ; \quad e_{0}=0,1 ; \quad a_{0}=5000 \mathrm{M} / \mathrm{c}\right)
$$

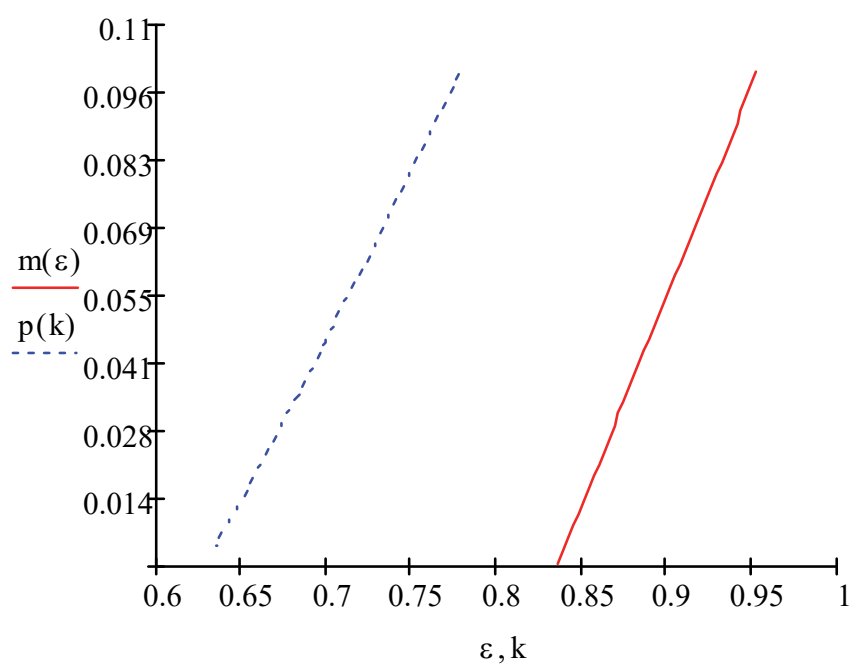

Figure. 5. The graph for dependence between quantities $\varepsilon, k$ and $f(\varepsilon), a(k)$ :

$$
\left(\gamma_{0}=\frac{\pi}{6} ; \quad e_{0}=0,1 ; \quad a_{0}=5000 \mathrm{M} / \mathrm{c}\right)
$$

Let the cylinder on border $s=0$ unload with a speed $v(t)$.

From (25)

$$
\vartheta(t)=-\int_{\varepsilon_{0}}^{\varepsilon} a\left(x_{s}\right) d x_{s} .
$$

Where $\vartheta$ is a function of the top limit of an integral.
The equation (26) is the equation for defining an axial deformation of a network $x_{s}=\varepsilon$ (unlike deformation of fibers $e$ ).

Approximately having presented integral (19) in the form of the sum, we have:

$$
\vartheta=-\int_{\varepsilon_{0}}^{\varepsilon} a(\eta) d \eta
$$


$\vartheta=\int_{\varepsilon}^{\varepsilon_{0}} a(\eta) d \eta$

$\vartheta_{0}=a\left(\varepsilon_{0}\right) \Delta \varepsilon$

$\vartheta_{1}=\left(a\left(\varepsilon_{0}\right)+a\left(\varepsilon_{1}\right)\right) \cdot \Delta \varepsilon$

$\vartheta_{2}=\left(a\left(\varepsilon_{0}\right)+a\left(\varepsilon_{1}\right)+a\left(\varepsilon_{2}\right)\right) \cdot \Delta \varepsilon$

$\vartheta_{n}=\left(a\left(\varepsilon_{0}\right)+a\left(\varepsilon_{1}\right)+\cdots+a\left(\varepsilon_{n}\right)\right) \cdot \Delta \varepsilon$ or $\vartheta=f(\varepsilon)$

inverse relationship $\varepsilon \rightarrow \vartheta$ on border. As positive characteristics are rectilinear, it is possible to define $\varepsilon$ in all area of movement. Functional dependence of speed of movement - speed of a wave and deformation for the given example is presented in the tables 1 and 2 .

$\vartheta_{n}=f\left(\varepsilon_{0}-\varepsilon\right)$.

Calculated values of the utilized parameters (for $\gamma_{0}=\frac{\pi}{4}$ )

\begin{tabular}{|c|c|c|c|c|c|c|c|c|c|c|c|}
\hline$\varepsilon_{0}$ & $\varepsilon_{1}$ & $\varepsilon_{2}$ & $\varepsilon_{3}$ & $\varepsilon_{4}$ & $\varepsilon_{5}$ & $\varepsilon_{6}$ & $\varepsilon_{7}$ & $\varepsilon_{8}$ & $\varepsilon_{9}$ & $\varepsilon_{10}$ & $\varepsilon_{11}$ \\
\hline 0.778 & 0.770 & 0.762 & 0.754 & 0.746 & 0.738 & 0.730 & 0.722 & 0.714 & 0.706 & 0.698 & 0.690 \\
\hline$e\left(\varepsilon_{0}\right)$ & $e\left(\varepsilon_{1}\right)$ & $e\left(\varepsilon_{2}\right)$ & $e\left(\varepsilon_{3}\right)$ & $e\left(\varepsilon_{4}\right)$ & $e\left(\varepsilon_{5}\right)$ & $e\left(\varepsilon_{6}\right)$ & $e\left(\varepsilon_{7}\right)$ & $e\left(\varepsilon_{8}\right)$ & $e\left(\varepsilon_{9}\right)$ & $e\left(\varepsilon_{10}\right)$ & $e\left(\varepsilon_{11}\right)$ \\
\hline 0.1 & 0.094 & 0.089 & 0.083 & 0.078 & 0.072 & 0.067 & 0.061 & 0.056 & 0.050 & 0.045 & 0.040 \\
\hline $9_{0}$ & $\vartheta_{1}$ & $9_{2}$ & $9_{3}$ & $9_{4}$ & 95 & $9_{6}$ & $9_{7}$ & 98 & 99 & $9_{10}$ & $\vartheta_{11}$ \\
\hline $747 \cdot 10^{3}$ & $1.493 \cdot 10^{3}$ & $2.237 \cdot 10^{3}$ & $2.980 \cdot 10^{3}$ & $3.721 \cdot 10^{3}$ & $4.461 \cdot 10^{3}$ & $5.199 \cdot 10^{3}$ & $5.936 \cdot 10^{3}$ & $6.672 \cdot 10^{3}$ & $7.406 \cdot 10^{3}$ & $8.139 \cdot 10^{3}$ & $8.87 \cdot 10^{3}$ \\
\hline$a\left(\varepsilon_{0}\right)$ & $a\left(\varepsilon_{1}\right)$ & $a\left(\varepsilon_{2}\right)$ & $a\left(\varepsilon_{3}\right)$ & $a\left(\varepsilon_{4}\right)$ & $a\left(\varepsilon_{5}\right)$ & $a\left(\varepsilon_{6}\right)$ & $a\left(\varepsilon_{7}\right)$ & $a\left(\varepsilon_{8}\right)$ & $a(\varepsilon 9)$ & $a\left(\varepsilon_{10}\right)$ & $a\left(\varepsilon_{11}\right)$ \\
\hline $7.753 \cdot 10^{4}$ & $7.732 \cdot 10^{4}$ & $7.711 \cdot 10^{4}$ & $7.689 \cdot 10^{4}$ & $7.667 \cdot 10^{4}$ & $7.644 \cdot 10^{4}$ & $7.621 \cdot 10^{4}$ & $7.598 \cdot 10^{4}$ & $7.575 \cdot 10^{4}$ & $7.551 \cdot 10^{4}$ & $7.526 \cdot 10^{4}$ & $7.502 \cdot 10^{4}$ \\
\hline
\end{tabular}

Calculated values of the utilized parameters (for $\gamma_{0}=\frac{\pi}{6}$ )

\begin{tabular}{|c|c|c|c|c|c|c|c|c|c|c|c|}
\hline$\varepsilon_{0}$ & $\varepsilon_{1}$ & $\varepsilon_{2}$ & $\varepsilon_{3}$ & $\varepsilon_{4}$ & $\varepsilon_{5}$ & $\varepsilon_{6}$ & $\varepsilon_{7}$ & $\varepsilon_{8}$ & $\varepsilon 9$ & $\varepsilon_{10}$ & $\varepsilon_{11}$ \\
\hline 0.953 & 0.950 & 0.947 & 0.944 & 0.941 & 0.938 & 0.935 & 0.932 & 0.929 & 0.926 & 0.923 & 0.920 \\
\hline$e\left(\varepsilon_{0}\right)$ & $e\left(\varepsilon_{1}\right)$ & $e\left(\varepsilon_{2}\right)$ & $e\left(\varepsilon_{3}\right)$ & $e\left(\varepsilon_{4}\right)$ & $e\left(\varepsilon_{5}\right)$ & $e\left(\varepsilon_{6}\right)$ & $e\left(\varepsilon_{7}\right)$ & $e\left(\varepsilon_{8}\right)$ & $e\left(\varepsilon_{9}\right)$ & $e\left(\varepsilon_{10}\right)$ & $e\left(\varepsilon_{11}\right)$ \\
\hline 0.230 & 0.228 & 0.225 & 0.223 & 0.221 & 0.219 & 0.216 & 0.214 & 0.212 & 0.209 & 0.207 & 0.205 \\
\hline $9_{0}$ & $\vartheta_{1}$ & $\boldsymbol{9}_{2}$ & $9_{3}$ & $\vartheta_{4}$ & $9_{5}$ & 96 & $9_{7}$ & 98 & 99 & $9_{10}$ & $\vartheta_{11}$ \\
\hline 866.7 & $1.732 \cdot 10^{3}$ & $2.597 \cdot 10^{3}$ & $3.461 \cdot 10^{3}$ & $4.324 \cdot 10^{3}$ & $5.186 \cdot 10^{3}$ & $6.048 \cdot 10^{3}$ & $6.908 \cdot 10^{3}$ & $7.767 \cdot 10^{3}$ & $8.626 \cdot 10^{3}$ & $9.483 \cdot 10^{3}$ & $1.034 \cdot 10^{3}$ \\
\hline$a\left(\varepsilon_{0}\right)$ & $a\left(\varepsilon_{1}\right)$ & $a\left(\varepsilon_{2}\right)$ & $a\left(\varepsilon_{3}\right)$ & $a\left(\varepsilon_{4}\right)$ & $a\left(\varepsilon_{5}\right)$ & $a\left(\varepsilon_{6}\right)$ & $a\left(\varepsilon_{7}\right)$ & $a(88)$ & $a(\varepsilon 9)$ & $a\left(\varepsilon_{10}\right)$ & $a\left(\varepsilon_{11}\right)$ \\
\hline $8.667 \cdot 10^{4}$ & $8.658 \cdot 10^{4}$ & $8.648 \cdot 10^{4}$ & $8.639 \cdot 10^{4}$ & $8.630 \cdot 10^{4}$ & $8.621 \cdot 10^{4}$ & $8.612 \cdot 10^{4}$ & $8.603 \cdot 10^{4}$ & $8.593 \cdot 10^{4}$ & $8.584 \cdot 10^{4}$ & $8.575 \cdot 10^{4}$ & $8.566 \cdot 10^{4}$ \\
\hline
\end{tabular}

\section{Conclusions}

Setting on border speed of movement of the end of a network as a time function it is possible to define deformation as time function on the end of a network and to the above-stated form everywhere in area $\mathrm{SOt}$.

For an example takes $\vartheta=b t$ then $t=f(\varepsilon) / b$.
Depending on distribution of speed on the border, deformation of a constant on characteristics is defined (figures 4 and 5).

\section{References}

1. Rakhmatulin Kh.A. (1947). Ob udare po gibkoi niti [On hitting a flexible thread]. Journal Appl. Math. Mech., XII, 379-382. (In Russ.) 
2. Agalarov J.H., Efendiev A.N. (1988). The propagation of nonlinear waves in a structure consisting of net system. Rakenteiden mekaniika seura ry. Finish association for structural mechanics, 21(2), 3-10.

3. Agalarov J.H., Gulieva (Rustamova) M.A. (1998). Movement equation of a net in the plane. Izv. AN Azerb. Mathematics and mechanics, XVIII(2), 103-105

4. Seyfullayev A.I., Gulieva (Rustamova) M.A. (2000). To the solution of the equilibrium problem of the net. Proceedings of Institute of Mathematics and Mechanics of AS of Azerbaijan, (XIII), 144-147.

5. Agalarov D.G., Seyfullaev A.I., Gulieva (Rustamova) M.A. (2001). Chislennoe reshenie odnoj ploskoj zadachi ravnovesiya seti [The numerical decision of one flat problem of balance of a network]. The Mechanic Engineering, (1), 3-4. (In Russ.)

6. Gulieva (Rustamova) M.A. (2002). Tension of a rectangular net fastened from two adjacent sides. Proceedings of Institute of Mathematics and Mechanics of NAS of Azerbaijan, XVI(XXIV), 156-160.

7. Agalarov J.H., Gulieva (Rustamova) M.A. (2002). Waves of strong breaks in nets. Proceedings of Institute of Mathematics and Mechanics of NAS of Azerbaijan, XVII (XXV), 135-137.
8. Agalarov J.H. (1982). Issledovanie dvizheniya setej pri udare [Research of movement of networks at blow]. News of Academy of Sciences of the Azerbaijan Soviet Socialist Republic. Series of Physicotechnical and Mathematical Sciences, (6), 38-41. (In Russ.)

9. Barenblat G.I. (1953). O rasprostranenii mgnovennyh vozmushchenij v srede s nelinejnoj zavisimost'yu napryazhenij ot deformacij [About distribution of instant indignations to the environment with nonlinear dependence of pressure on deformations]. PMM, XVII(4). (In Russ.)

\section{About the author}

Mexseti A. Rustamova - PhD in Physical and Mathematical Sciences, Leading Researcher, Associate Professor, Department of Wave Dynamics, Institute of Mathematics and Mechanics, National Academy of Sciences of Azerbaijan. Research interests: the wave dynamics. Contacts: e-mail-mehsetir@gmail.com

\section{For citation}

Rustamova M.A. (2019). Unloading wave in the cylindrical network from nonlinear elastic fibers. Structural Mechanics of Engineering Constructions and Buildings, 15(2), 149-157. DOI: 10.22363/1815-5235-2019-15-2-149-157

НАУЧНАЯ СТАТЬЯ

\section{Волна разгрузки в цилиндрической сети из нелинейно упругих волокон}

\section{М.А. Рустамова}

Институт математики и механики Национальной академии наук Азербайджана, Азербайджанская Республика, АZ1143, Баку, ул. Б. Вахабзаде, 9

Поступила в редакциию: 30 января 2019 г. Доработана: 27 февраля 2019 г.

Принята к публикации: 16 марта 2019 г.

Ключевые слова:

нелинейно упругие волокна; волна разгрузки; цилиндрическая сеть; непрерывные волны

\section{Аннотация}

Цели. Исследование волны разгрузки в цилиндрической сети из нелинейно упругих волокон. Предпринимается попытка решения задачи о непрерывных волнах с учетом множества вариантов распространения волн в цилиндрических сетях.

Memodb. На основе уравнений движения сети в общем случае строятся уравнения движения цилиндрической сети. Рассматривается движение сети в осевом направлении. За базис цилиндрической системы принимаются: единичный вектор $\vec{i}$, параллельный оси цилиндра, $\vec{j}-$ единичный вектор касательной к поперечному сечению цилиндра, $\vec{k}-$ единичный вектор, перпендикулярный к предыдущим, $x$ - координата в направлении оси цилиндра, $y$ - длина дуги окружности цилиндра. Задача сводится к гиперболической системе уравнений при соответствующих условиях.

Поскольку при растяжении сети скорость волны увеличивается, то, очевидно, волна растяжения будет разрывной. С целью исследования непрерывных волн решается задача о распространении волн при разгрузке предварительно растянутого цилиндра из нелинейной основы. Задача решается методом характеристик.

Результаты. Результаты иллюстрируются расчетами и могут быть использованы при вычислении данных для различных гибких труб, в том числе бурильных. 


\section{Список литературы}

1. Рахматулин Х.А. Об ударе по гибкой нити // ПММ. 1947. Т. XII. С. 379-382.

2. Agalarov J.H., Efendiev A.N. The propagation of nonlinear waves in a structure consisting of net system // Rakenteiden mekaniika seura ry. Finish association for structural mechanics. 1988. Vol. 21. No. 2. Pp. 3-10.

3. Agalarov J.H., Gulieva (Rustamova) M.A. Movement equation of a net in the plane // Изв. АН Азерб. Сер. физ.мат. наук. Математика и механика. 1998. Т. XVIII. № 2. C. 103-105.

4. Seyfullayev A.I., Gulieva (Rustamova) M.A. To the solution of the equilibrium problem of the net // Proceedings of Institute of Mathematics and Mechanics of AS of Azerbaijan. 2000. Vol. XIII. Pp. 144-147.

5. Агаларов Д.Г., Сейфуллаев А.И., Гулиева (Рустамова) М.A. Численное решение одной плоской задачи равновесия сети // Механика машиностроение. 2001. № 1. C. 3-4.

6. Gulieva (Rustamova) M.A. Tension of a rectangular net fastened from two adjacent sides // Proceedings of Institute of Mathematics and Mechanics of NAS of Azerbaijan. 2002. Vol. XVI (XXIV). Pp. 156-160.

7. Agalarov J.H., Gulieva (Rustamova) M.A. Waves of strong breaks in nets // Proceedings of Institute of
Mathematics and Mechanics of NAS of Azerbaijan. 2002. Vol. XVII (XXV). Pp. 135-137.

8. Агаларов Д.Г. Исследование движения сетей при ударе // Известия Академии наук Азербайджанской ССР. Серия физико-технических и математических наук. 1982. № 6. C. 38-41.

9. Баренблат Г.И. О распространении мгновенных возмущений в среде с нелинейной зависимостью напряжений от деформаций // ПММ. 1953. Т. XVII. № 4.

\section{Об авторе}

Рустамова Мехсети Акиф кызы - кандидат физико-математических наук, ведущий научный сотрудник, доцент, отдел волновой динамики, Институт математики и механики, Национальная академия наук Азербайджана. Область научных интересов: волновая динамика. Контактная информация: e-mail - mehsetir@ gmail.com

\section{Для цитирования}

Rustamova M.A. Unloading wave in the cylindrical network from nonlinear elastic fibers (Волна разгрузки в цилиндрической сети из нелинейно упругих волокон) // Строительная механика инженерных конструкций и сооружений. 2019. Т. 15. № 2. C. 149-157. DOI: 10.22363/ 1815-5235-2019-15-2-149-157 